\title{
CTLA-4 Gene Polymorphism and the Risk of Systemic Lupus Erythematosus in the Chinese Population
}

\author{
Ahmad Taha Khalaf, ${ }^{1}$ Ji-Quan Song, ${ }^{2}$ Ting-Ting Gao, ${ }^{2}$ Xiang-Ping Yu, ${ }^{2}$ and Tie-Chi Lei ${ }^{1}$ \\ ${ }^{1}$ Renmin Hospital of Wuhan University, Wuhan 430060, China \\ ${ }^{2}$ Zhongnan Hospital of Wuhan University, Wuhan 430071, China
}

Correspondence should be addressed to Ji-Quan Song, songjiquan@tom.com and Tie-Chi Lei, tchlei@whu.edu.cn

Received 14 April 2011; Revised 25 May 2011; Accepted 9 June 2011

Academic Editor: Nathan A. Ellis

Copyright (C) 2011 Ahmad Taha Khalaf et al. This is an open access article distributed under the Creative Commons Attribution License, which permits unrestricted use, distribution, and reproduction in any medium, provided the original work is properly cited.

\begin{abstract}
Several variants of CTLA-4 have been reported to be associated with susceptibility systemic lupus erythematosus (SLE); however, findings have been inconsistent across different populations. Using a case-control study design, we have investigated the role of CTLA-4 polymorphism at positions -1661 and -1722 on SLE susceptibility in our Chinese SLE population in central China's Hubei province. Samples were collected from 148 SLE patients and 170 healthy controls. Polymerase chain reaction restriction fragments length polymorphism (PCR-RFLP) was used to analyze the genotypes of the two sites. Statistically significant difference was observed in genotypes for -1722 , but not for -1661 . The frequency of the $\mathrm{T}$ allele on the -1722 SNP was significantly increased in SLE patients: $57.8 \%$ versus $40.6 \%$ in controls $(P<0.001, \mathrm{OR}=2.002)$. While the detected $\mathrm{C}$ allele frequency in the controls was significantly elevated in comparison to that in the SLE patients (59.4\% versus $42.2 \%)$. On the contrary, no association was found between SLE and CTLA-4 polymorphism at position - 1661 .
\end{abstract}

\section{Introduction}

Systemic lupus erythematosus (SLE) is a chronic multisystem autoimmune disease and is considered to be caused by complex interactions between genetic risk, environmental, and hormonal factors that result in an immune dysregulation, and autoantibody production ensued [1-3]. Epidemiological studies reported that SLE is more common in Asians (46.7/100 000) than in Caucasians (20.7/100 000), and ethnicity also influences the age of onset and severity of its manifestations $[4,5]$.

The precise aetiopathogenesis of SLE is still unclear; however, the search for genes and molecular interactions that influence disease has promoted our understanding of pathogenesis and genetic contributions to autoimmunity in SLE [6]. Genetic association studies and recent advances in the field of single nucleotide polymorphisms (SNPs) have been highly successful in identifying several loci associated with disease susceptibility $[7,8]$.

The CTLA-4 molecule has a suppressive effect on Tcell activation and might contribute to maintain immune tolerance by blocking CD28-dependent $\mathrm{T}$ cell activation through interactions with its ligand CD80/86 on antigen presenting cells [7]. The CTLA-4/B7 complex can compete with the CD28/B7 complex and convey an inhibitory influence to the $\mathrm{T}$ cell affecting $\mathrm{T}$ cell development, cytokine production, and immune reactions [8].

CTLA-4 would, therefore, be an important negative regulator of T-cell responses, and its dysregulation has the potential to affect the pathogenesis of SLE by altered activation of $\mathrm{T}$ cells to self-antigens $[9,10]$. The CTLA-4 gene is located within the risk region on chromosome $2 \mathrm{q} 33$, and several polymorphisms have been reported in this gene. However, only few of them have been studied for association with SLE susceptibility, of which, two are located within the promoter region: a T/C change at position -1722 and an A/G transition at position -1661 [11-13]. The former could alter transcription factor binding sites whereas the latter may alter the potential response element for myocyte enhancer factor 2 (MEF2) [14]. Hence, allelic variations of these two sites might lead to a differential susceptibility to SLE resulting from unbalanced or inefficient immune responses. 
Although CTLA-4 polymorphism has been shown to be associated with a number of autoimmune diseases, including SLE, Graves' disease, multiple sclerosis, and type 1 diabetes, the associations have not been always replicated in different populations $[11,12,15]$. Recent studies showed that the CTLA-4 polymorphism plays an important role in SLE in some populations, which has not been confirmed in Chinese. Using a case-control study design, we have investigated the role of CTLA-4 polymorphism at positions -1661 and -1722 on SLE susceptibility in our Chinese SLE population in central China's Hubei Province.

\section{Patients and Methods}

2.1. Study Population. A total of 148 patients (17 males and 131 females) meeting the 1997 revised criteria of the American College of Rheumatology (ACR) for SLE [16] were recruited from Renmin and Zhongnan Hospitals of Wuhan University, Wuhan, China. Controls were 170 healthy volunteers with no history of autoimmune disease, collected for a case-control study. All patients and controls were Han Chinese residing in the central part of China. The study was approved by the hospitals' Ethics Committee of Wuhan University, and all subjects were consented to participate in the study.

2.2. DNA Extraction and Genotyping. DNA from patients and controls was extracted from peripheral blood with DNA flash kit 2.0 (HaiGene Biotechnolgy Co., Ltd., Gentra Systems Corp.) according to the standard protocol from the manufacturer. The polymorphisms at positions -1661 and -1722 were analyzed by PCR-RFLP (polymerase chain reaction-restriction fragments length polymorphism) [13], using the specific oligonucleotide primers (Sangon, Shanghai, China), 5'CTAAGAGCATCCGCTTGCACCT3' and 5'TTGGTGTGATGCACAGAAGCCTTTT3'. PCR amplification conditions were carried out as follows: initial denaturation at $94^{\circ} \mathrm{C}$ for 5 minutes, then thirty cycles at $94^{\circ} \mathrm{C}(15 \mathrm{~s}), 60^{\circ} \mathrm{C}(30 \mathrm{~s}), 72^{\circ} \mathrm{C}(45 \mathrm{~s})$, and one final extension at $72^{\circ} \mathrm{C}$ for $8 \mathrm{~min}$. The products of the PCR were digested with $B v b \mathrm{I}$ or $\mathrm{MseI}$ at $37^{\circ} \mathrm{C}$ for $4 \mathrm{~h}$, and then were analyzed by $2 \%$ agarose gel electrophoresis stained with ethidium bromide. After resolving, the $-1722 \mathrm{~T} / \mathrm{C}$ polymorphism was determined by detecting a 486 bp digested fragment ( $\mathrm{T}$ allele) or two fragments of 270 and $216 \mathrm{bp}$ (C allele); (Figure 1). The $-1661 \mathrm{~A} / \mathrm{G}$ polymorphism was determined by detecting a $486 \mathrm{bp}$ fragment ( $\mathrm{G}$ allele) or two fragments of 347 and 139 bp (A allele); (Figure 2).

2.3. Statistical Analyses. We tested for Hardy-Weinberg equilibrium (HWE) among cases and controls. Allelic and genotypic frequencies were calculated by direct counting. The chi-square test with Yates correction and Fisher exact test were used to compare genotypes and alleles frequencies. Statistical significance was defined as $P<0.05$. The odds ratio $(\mathrm{OR})$ was calculated to measure the strength of the association observed.

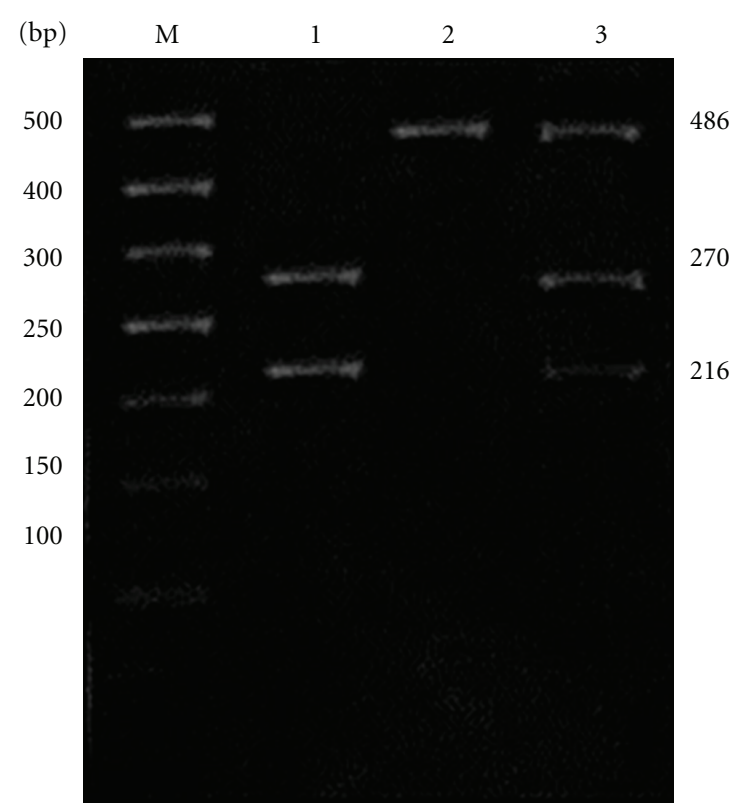

FIGURE 1: PCR restriction fragment length polymorphism results of $-1722 \mathrm{~T}$ to $\mathrm{C}$ substitution in CTLA-4 promoter region. (1) CC genotype; (2) TT genotype; (3) TC genotype.

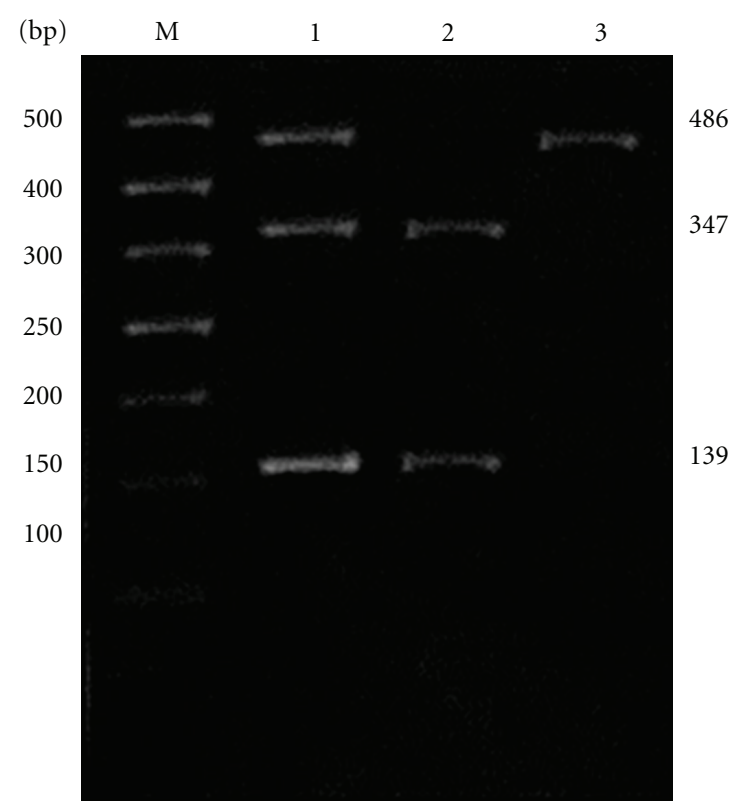

FIGURE 2: PCR restriction fragment length polymorphism results of -1661 A to $G$ substitution in CTLA-4 promoter region.(1) AG genotype; (2) AA genotype; (3) GG genotype.

\section{Results and Discussion}

The genotypic frequencies for the two sites tested were not found to be deviated from those predicted from HardyWeinberg equilibrium in both SLE patients and controls. Genotype and allele frequencies of the $-1722 \mathrm{~T} / \mathrm{C}$ and $-1661 \mathrm{~A} / \mathrm{G}$ polymorphisms are shown in Tables 1 and 2. As observed, the genotypes at position -1722 were strongly 
TABLE 1: Genotypic distribution and allelic frequencies of - 1722 CTLA-4 polymorphisms in Chinese SLE patients and healthy controls.

\begin{tabular}{|c|c|c|c|c|c|}
\hline Promoter -1722 & $\begin{array}{c}\text { SLE } \\
n=148\end{array}$ & $\begin{array}{c}\text { Controls } \\
n=170\end{array}$ & $x^{2}$ & $P$ value & OR (95\% CI) \\
\hline \multicolumn{6}{|c|}{ Genotype frequency } \\
\hline $\mathrm{TT}$ & $42(28.4 \%)$ & $29(17.1 \%)$ & 5.846 & 0.016 & $1.926(1.127-3.293)$ \\
\hline $\mathrm{TC}$ & $87(58.8 \%)$ & $80(47.1 \%)$ & 4.362 & 0.037 & $1.605(1.028-2.503)$ \\
\hline $\mathrm{CC}$ & $19(12.8 \%)$ & $61(35.8 \%)$ & 22.315 & $<0.001$ & $0.263(0.148-0.468)$ \\
\hline \multicolumn{6}{|l|}{ Allele frequency } \\
\hline $\mathrm{T}$ & $171(57.8 \%)$ & $138(40.6 \%)$ & 18.701 & $<0.001$ & $2.002(1.459-2.747)$ \\
\hline $\mathrm{C}$ & $125(42.2 \%)$ & $202(59.4 \%)$ & & & \\
\hline \multicolumn{6}{|l|}{ Phenotype } \\
\hline $\mathrm{T}$ & $129(87.2 \%)$ & $109(64.1 \%)$ & 6.182 & 0.013 & $1.574(1.100-2.253)$ \\
\hline $\mathrm{C}$ & $106(71.6 \%)$ & $141(82.9 \%)$ & & & \\
\hline
\end{tabular}

Abbreviations: CTLA-4 = cytotoxic T-lymphocyte antigen 4; SLE =systemic lupus erythematosus; OR = odds ratios; $95 \% \mathrm{CI}=95 \%$ confidence interval.

TABLE 2: Genotypic distribution and allelic frequencies of - 1661 CTLA-4 polymorphisms in Chinese SLE patients and healthy controls.

\begin{tabular}{|c|c|c|c|c|c|}
\hline Promoter -1661 & $\begin{array}{c}\text { SLE } \\
n=148\end{array}$ & $\begin{array}{c}\text { Controls } \\
n=170\end{array}$ & $x^{2}$ & $P$-value & OR (95\% CI) \\
\hline \multicolumn{6}{|c|}{ Genotype frequency } \\
\hline $\mathrm{AA}$ & $59(39.9 \%)$ & $63(37.1 \%)$ & 0.263 & 0.608 & $1.126(0.716-1.771)$ \\
\hline AG & $67(45.2 \%)$ & $76(44.7 \%)$ & 0.010 & 0.920 & $1.132(0.730-1.755)$ \\
\hline GG & $22(14.9 \%)$ & $31(18.2 \%)$ & 0.647 & 0.421 & $0.783(0.431-1.423)$ \\
\hline \multicolumn{6}{|l|}{ Allele frequency } \\
\hline A & $185(62.5 \%)$ & $202(59.4 \%)$ & 0.633 & 0.426 & $1.139(0.827-1.568)$ \\
\hline G & $111(37.5 \%)$ & $138(40.6 \%)$ & & & \\
\hline \multicolumn{6}{|l|}{ Phenotype } \\
\hline A & $126(85.1 \%)$ & $139(81.8 \%)$ & 0.207 & 0.649 & $1.090(0.752-1.578)$ \\
\hline G & $89(60.1 \%)$ & $107(62.9 \%)$ & & & \\
\hline
\end{tabular}

Abbreviations: CTLA-4 = cytotoxic T-lymphocyte antigen 4; SLE = systemic lupus erythematosus; OR = odds ratios; $95 \%$ CI = 95\% confidence interval.

associated with SLE. The frequency of the T allele on the -1722 SNP was significantly increased in SLE patients: $57.8 \%$ versus $40.6 \%$ in controls $(P<0.001$, OR $=2.002)$. While the detected $\mathrm{C}$ allele frequency in the controls was significantly elevated in comparison with that in the SLE patients $(59.4 \%$ versus $42.2 \%)$. The frequencies of $\mathrm{T} / \mathrm{T}$ homozygotes and T/C heterozygotes were also significantly higher in patients than in controls $(28.4 \%$ versus $17.1 \%$, $P=0.016, \mathrm{OR}=1.926 ; 58.8 \%$ versus $47.1 \%, P=0.037, \mathrm{OR}=$ 1.605). Conversely, the frequencies of $\mathrm{C} / \mathrm{C}$ homozygotes was considerably higher in controls than in patients $(35.8 \%$ versus $12.8 \%, P<0.01, \mathrm{OR}=0.263)$. We observed no significant difference in the distribution of the alleles and genotypes for the -1661 site between patients and healthy subjects.

Notwithstanding the convincing evidence that CTLA-4 polymorphism plays an important role in susceptibility to SLE, contradictory result has been reported among different populations [12]. Several studies have observed a significant association of SLE with CTLA-4 gene polymorphisms [12, 13, 17-19]. While on the contrary, other studies showed a lack of association with that genetic variation [20-22]. Reasons for the variability in associations are still ambiguous. Thus, investigating the frequencies and distribution of variants CTLA-4 gene across populations are essential for understanding disease association and discovery of population differences, especially for Chinese as they have a much higher SLE prevalence than the Europeans $[4,5]$. The interval encompassing the CTLA-4 locus on chromosome $2 \mathrm{q} 33$ has been reported to show linkage to SLE in two genome-wide association studies (GWAS) [23, 24]. A very recent GWAS has confirmed that variation in the CTLA-4 gene has been associated with a genetic risk of SLE [25]. Although, this polymorphism may has not yet reached stringent thresholds of lupus GWAS performed in some Chinese cohorts, however, the association has been observed in multiple independent studies in various ethnic populations including Asian [12, 13, 17-19, 23-26], and there is strong biological evidence sufficient to conclude that CTLA-4 polymorphism confers susceptibility to SLE through its crucial functions in $\mathrm{T}$ cell activation/regulation $[11,24,25,27-30]$.

Recently, a GWAS study confirmed an SLE susceptibility locus at chromosome $2 \mathrm{q} 32.3$ in Chinese population that is near the region $2 \mathrm{q} 33$ which encodes the genes for CTLA-4 [31]. As polymorphisms that are near each other have 
a tendency to be inherited together, something worth further pursuit in future studies. In Asian people, positive associations of the CTLA-4 polymorphism with SLE were reported in Korean [13] and Japanese [17] while no such association was found in Malaysian population [32]. Significantly, a meta-analysis by Lee et al. found a close association between SLE and exon 1 at +49 of CTLA- 4 gene, especially in Asians [12]. Liu et al. did not observe an association of CTLA-4 polymorphism with SLE in Taiwanese, but suggested that it is possible that this polymorphism could affect some specific clinical features [20]. This discrepancy with the findings of the current study may be partially attributed to the variations among Chinese living in different geographical regions [33].

The results of this study confirm the involvement of CTLA-4 polymorphisms at the promoter -1722 on SLE susceptibility in the Chinese population. Our findings are in agreement with the results of other ethnic groups demonstrating the influence of this polymorphism in the susceptibility to SLE. On the contrary, the genotypic frequencies for the -1661 site were not found to be significantly different between patients and controls. Other studies on -1661 polymorphism in Korean with SLE also could not find a positive correlation [13]. In addition, no overall associations were seen between this polymorphism and SLE in African-Americans [22]. Being inexplicit, despite the short distance between the two locations, only -1722 Promoter region polymorphisms was associated with SLE, while the other -1661 is not. This possibly due to some functional differences between the two sites on regulatory properties of this promoter might affect basal promoter activity and gene expression. Polymorphisms in promoter regions may affect the gene expression quantitatively or qualitatively by altering transcription factor binding sites or other controlling domains [34].

The significant increase in the $\mathrm{C}$ allele observed in the controls is assumed to play a protective role against SLE in Chinese. On the other hand, The T/T and T/C genotypes of $-1722 \mathrm{~T} / \mathrm{C}$ polymorphisms were associated with higher risk to have SLE. Hudson et al. [13] studied CTLA-4 polymorphisms at positions T/C -1722 in Korean population and found that $\mathrm{T}$ allele was more frequent in SLE patients while $\mathrm{C}$ allele was decreased in the controls and suggested that the $\mathrm{C}$ allele could contribute protectively to SLE in the Korean population. In contrast, Fernandez-Blanco et al. [18] found that the C allele of the -1722 T/C SNP was associated with SLE susceptibility in the Spanish SLE patients. However, another study did not find any significant association between the genetic polymorphisms at the -1722 T/C SNP of the CTLA-4 gene and Spanish SLE patients [21]. Although there were significant associations in two of the three studies described above. However, a published meta-analysis study did not unveil a significantly increased odds ratio for this polymorphism [12]. Takeuchi et al. also reported a slight increase in the allele frequency of $-1722 \mathrm{C}$ in Japanese patients with SLE compared to the controls, but the difference was not statistically significant [35].

Because there are relatively few studies, it is difficult to explain the contradictories at the promoter -1722 polymorphism in SLE, but they may be due to a different genetic background and a possible role for racial and ethnic influences in the pattern of haplotypes on the CTLA-4 locus between various populations in SLE predisposition $[4,5]$. Variations in associations among populations may be also related to difference in patient characteristics or the distribution of other risk factors that interact with CTLA-4 concerning SLE. Moreover, genetic evidence suggests that genetic heterogeneity, a common phenomenon in complex diseases, are responsible for a considerable portion of this variability [36]. We believe that there is not sufficient research on CTLA4 polymorphisms to identify and demonstrate the role of these important genetic variants that confer susceptibility to SLE susceptibility among different populations.

Whereas some polymorphisms on the CTLA-4 gene have been analyzed in various populations for investigating an association with SLE, only one of these, the CTLA-4 A/G polymorphism at position +49 in exon 1 , has been widely studied, mainly in European populations [12]. Accordingly, we expect that the results of our study to reinforce the interest of focusing on analyzing the role of CTLA-4 polymorphism in increased susceptibility to SLE. Furthermore, an elevated level of soluble CTLA-4 in sera has been described in SLE, with a positive and significant correlation between plasma sCTLA-4 concentration and SLE activity [28, 29]. Besides, the therapeutic use of CTLA4Ig which is a soluble fusion protein that interferes with $\mathrm{T}$ cell activation by inhibiting the B7/CD28 costimulatory interaction, appears to delay or extenuate disease development in experimental models of lupus [37]. In this way, the results observed should provide new postulates for the immunological role of this costimulatory molecule in the pathogenesis of SLE and should facilitate recent advances in the exploration of therapeutic agents targeting T-cell activation in this disease [29].

Other polymorphisms have also been described in the promoter region ( -658 and -318$)$, the CT60 (A/G), and at the 3 -end of the gene as well as the microsatellite (AT)(n) in the $3^{\prime}$-untranslated region $\left(3^{\prime}\right.$-UTR) of the CTLA-4 gene. Nevertheless, the reported results have been inconsistent across different ethnic populations $[12,38]$. Hence, further explorations of these polymorphisms are needed in order to more fully examine SLE associations with CTLA-4 locus in Chinese population. Allelic and genotypic frequencies may vary between the populations; therefore, disease association studies and interpreting their results offer a possible route to understanding the influence of these genetic variants on disease aetiology and potentially to the development of new treatments.

\section{Conclusions}

CTLA-4 polymorphism at positions -1722 was significantly associated with SLE and may be a risk factor for SLE susceptibility in Chinese. Our results concur with the majority of those published supporting the important influence of CTLA-4 polymorphisms in the susceptibility to SLE. Nevertheless, further study in terms of the functional analysis of polymorphisms on the CTLA-4 gene needs to be done, and larger population studies in different ethnic groups should be performed in the future. 


\section{Acknowledgments}

This study was supported by Department of Dermatology, Renmin and Zhongnan hospitals of Wuhan University. The authors would like to thank Dr. Ahmed N. Abdalla for his kind help. Special thanks and appreciation are extended to our laboratory colleagues for preparing samples.

\section{References}

[1] J. C. Edberg, J. Wu, C. D. Langefeld et al., "Genetic variation in the CRP promoter: association with systemic lupus erythematosus," Human Molecular Genetics, vol. 17, no. 8, pp. 1147$1155,2008$.

[2] V. C. Kyttaris, S. Krishnan, and G. C. Tsokos, "Systems biology in systemic lupus erythematosus: integrating genes, biology and immune function," Autoimmunity, vol. 39, no. 8, pp. 705709, 2006.

[3] A. H. Sawalha, K. M. Kaufman, J. A. Kelly et al., "Genetic association of interleukin-21 polymorphisms with systemic lupus erythematosus," Annals of the Rheumatic Diseases, vol. 67, no. 4, pp. 458-461, 2008.

[4] C. S. Lau, G. Yin, and M. Y. Mok, "Ethnic and geographical differences in systemic lupus erythematosus: an overview," Lupus, vol. 15, no. 11, pp. 715-719, 2006.

[5] V. Rus and M. C. Hochberg, "The epidemiology of systemic lupus erythematosus," in Dubois' Lupus Erythematosus, D. J. Wallace and B. H. Hahn, Eds., pp. 65-83, Lippincott Williams \& Wilkins, Baltimore, Md, USA, 2002.

[6] I. Sekigawa, T. Naito, K. Hira et al., "Possible mechanisms of gender bias in SLE: a new hypothesis involving a comparison of SLE with atopy," Lupus, vol. 13, no. 4, pp. 217-222, 2004.

[7] J. B. Harley, J. A. Kelly, and K. M. Kaufman, "Unraveling the genetics of systemic lupus erythematosus," Springer Seminars in Immunopathology, vol. 28, no. 2, pp. 119-130, 2006.

[8] P. K. Gregersen and L. M. Olsson, "Recent advances in the genetics of autoimmune disease," Annual Review of Immunology, vol. 27, pp. 363-391, 2009.

[9] T. L. Walunas, C. Y. Bakker, and J. A. Bluestone, "CTLA-4 ligation blocks CD28-dependent T cell activation," Journal of Experimental Medicine, vol. 183, no. 6, pp. 2541-2550, 1996.

[10] B. M. Carreno and M. Collins, "The B7 family of ligands and its receptors: new pathways for costimulation and inhibition of immune responses," Annual Review of Immunology, vol. 20, pp. 29-53, 2002.

[11] H. Ueda, J. M. M. Howson, L. Esposito et al., "Association of the T-cell regulatory gene CTLA4 with susceptibility to autoimmune disease," Nature, vol. 423, no. 6939, pp. 506-511, 2003.

[12] Y. H. Lee, J. B. Harley, and S. K. Nath, "CTLA-4 polymorphisms and systemic lupus erythematosus (SLE): a metaanalysis," Human Genetics, vol. 116, no. 5, pp. 361-367, 2005.

[13] L. L. Hudson, K. Rocca, Y. W. Song, and J. P. Pandey, "CTLA4 gene polymorphisms in systemic lupus erythematosus: a highly significant association with a determinant in the promoter region," Human Genetics, vol. 111, no. 4-5, pp. 452455, 2002.

[14] V. Ling, P. W. Wu, H. F. Finnerty, A. H. Sharpe, G. S. Gray, and M. Collins, "Complete sequence determination of the mouse and human CTLA-4 gene loci: cross-species DNA sequence similarity beyond exon borders," Genomics, vol. 60, no. 3, pp. 341-355, 1999.
[15] O. P. Kristiansen, Z. M. Larsen, and F. Pociot, "CTLA-4 in autoimmune diseases-A general susceptibility gene to autoimmunity?" Genes and Immunity, vol. 1, no. 3, pp. 170$184,2000$.

[16] M. C. Hochberg, "Updating the American College of Rheumatology revised criteria for the classification of systemic lupus erythematosus," Arthritis and Rheumatism, vol. 40, no. 9, p. 1725, 1997.

[17] S. Ahmed, K. Ihara, S. Kanemitsu et al., "Association of CTLA4 but not CD28 gene polymorphisms with systemic lupus erythematosus in the Japanese population," Rheumatology, vol. 40, no. 6, pp. 662-667, 2001.

[18] L. Fernandez-Blanco, E. Perez-Pampin, J. J. Gomez-Reino, and A. Gonzalez, "A CTLA-4 polymorphism associated with susceptibility to systemic lupus erythematosus," Arthritis and Rheumatism, vol. 50, no. 1, pp. 328-329, 2004.

[19] M. Ulker, V. Yazisiz, N. Sallakci et al., "CTLA-4 gene polymorphism of exon $1(+49 \mathrm{~A} / \mathrm{G})$ in Turkish systemic lupus erythematosus patients," International Journal of Immunogenetics, vol. 36, no. 4, pp. 245-250, 2009.

[20] M. F. Liu, C. R. Wang, L. C. Lin, and C. R. Wu, "CTLA-4 gene polymorphism in promoter and exon-1 regions in Chinese patients with systemic lupus erythematosus," Lupus, vol. 10, no. 9, pp. 647-649, 2001.

[21] F. Aguilar, B. Torres, J. Sánchez-Román, A. Núñez-Roldán, and M. F. González-Escribano, "CTLA4 polymorphism in Spanish patients with systemic lupus erythematosus," Human Immunology, vol. 64, no. 10, pp. 936-940, 2003.

[22] C. G. Parks, L. L. Hudson, G. S. Cooper et al., "CTLA-4 gene polymorphisms and systemic lupus erythematosus in a population-based study of whites and African-Americans in the southeastern United States," Lupus, vol. 13, no. 10, pp. 784-791, 2004.

[23] R. M. Cantor, J. Yuan, S. Napier et al., "Systemic lupus erythematosus genome scan: support for linkage at 1q23, 2q33, 16q12-13, and 17q21-23 and novel evidence at 3p24, 10q2324, 13q32, and 18q22-23," Arthritis and Rheumatism, vol. 50, no. 10, pp. 3203-3210, 2004.

[24] D. S. C. Graham, A. K. Wong, N. J. McHugh, J. C. Whittaker, and T. J. Vyse, "Evidence for unique association signals in SLE at the CD28-CTLA4-ICOS locus in a family-based study," Human Molecular Genetics, vol. 15, no. 21, pp. 3195-3205, 2006.

[25] M. L. Budarf, P. Goyette, G. Boucher et al., "A targeted association study in systemic lupus erythematosus identifies multiple susceptibility alleles," Genes and Immunity, vol. 12, no. 1, pp. 51-58, 2010.

[26] M. Barreto, E. Santos, R. Ferreira et al., "Evidence for CTLA4 as a susceptibility gene for systemic lupus erythematosus," European Journal of Human Genetics, vol. 12, no. 8, pp. 620626, 2004.

[27] L. M. Gómez Osorio, J. Martín Ibañez, and J. M. Anaya Cabrera, "Autoimmunity co-signaling system: Regulatory $\mathrm{T}$ cells, CTLA-4 and FOXP3," Inmunología, vol. 12, no. 8, pp. 620-626, 2004.

[28] C. K. Wong, L. C. W. Lit, L. S. Tam, E. K. Li, and C. W. K. Lam, "Aberrant production of soluble costimulatory molecules CTLA-4, CD28, CD80 and CD86 in patients with systemic lupus erythematosus," Rheumatology, vol. 44, no. 8, pp. 989-994, 2005.

[29] M. F. Liu, C. R. Wang, P. C. Chen, and L. L. Fung, "Increased expression of soluble cytotoxic T-lymphocyte-associated 
antigen-4 molecule in patients with systemic lupus erythematosus," Scandinavian Journal of Immunology, vol. 57, no. 6, pp. 568-572, 2003.

[30] A. K. B. Lindqvist, K. Steinsson, B. Johanneson et al., "A susceptibility locus for human systemic lupus erythematosus (hSLE1) on chromosome 2q," Journal of Autoimmunity, vol. 14, no. 2, pp. 169-178, 2000.

[31] J. W. Han, H. F. Zheng, Y. Cui et al., "Genome-wide association study in a Chinese Han population identifies nine new susceptibility loci for systemic lupus erythematosus," Nature Genetics, vol. 41, no. 11, pp. 1234-1237, 2009.

[32] K. H. Chua, S. M. Puah, C. H. Chew, S. Y. Tan, and L. H. Lian, "Study of the CTLA-4 gene polymorphisms in systemic lupus erythematosus (SLE) samples from Malaysia," Annals of Human Biology, vol. 37, no. 2, pp. 274-280, 2010.

[33] S. Xu, X. Yin, S. Li et al., "Genomic dissection of population substructure of han Chinese and its implication in association studies," American Journal of Human Genetics, vol. 85, no. 6, pp. 762-774, 2009.

[34] B. S. Shastry, "SNP alleles in human disease and evolution," Journal of Human Genetics, vol. 47, no. 11, pp. 561-566, 2002.

[35] F. Takeuchi, S. Kuwata, and M. Mori, "CTLA-4-1661A/G and -1772 T/C dimorphisms in Japanese patients with systemic lupus erythematosus," Journal of Rheumatology, vol. 32, no. 10, pp. 2062-2063, 2005.

[36] A. E. Wandstrat, C. Nguyen, N. Limaye et al., "Association of extensive polymorphisms in the SLAM/CD2 gene cluster with murine lupus," Immunity, vol. 21, no. 6, pp. 769-780, 2004.

[37] M. Dall'Era and J. Davis, "CTLA4Ig: a novel inhibitor of costimulation," Lupus, vol. 13, no. 5, pp. 372-376, 2004.

[38] B. Torres, F. Aguilar, E. Franco et al., "Association of the CT60 marker of the CTLA4 gene with systemic lupus erythematosus," Arthritis and Rheumatism, vol. 50, no. 7, pp. 2211-2215, 2004. 

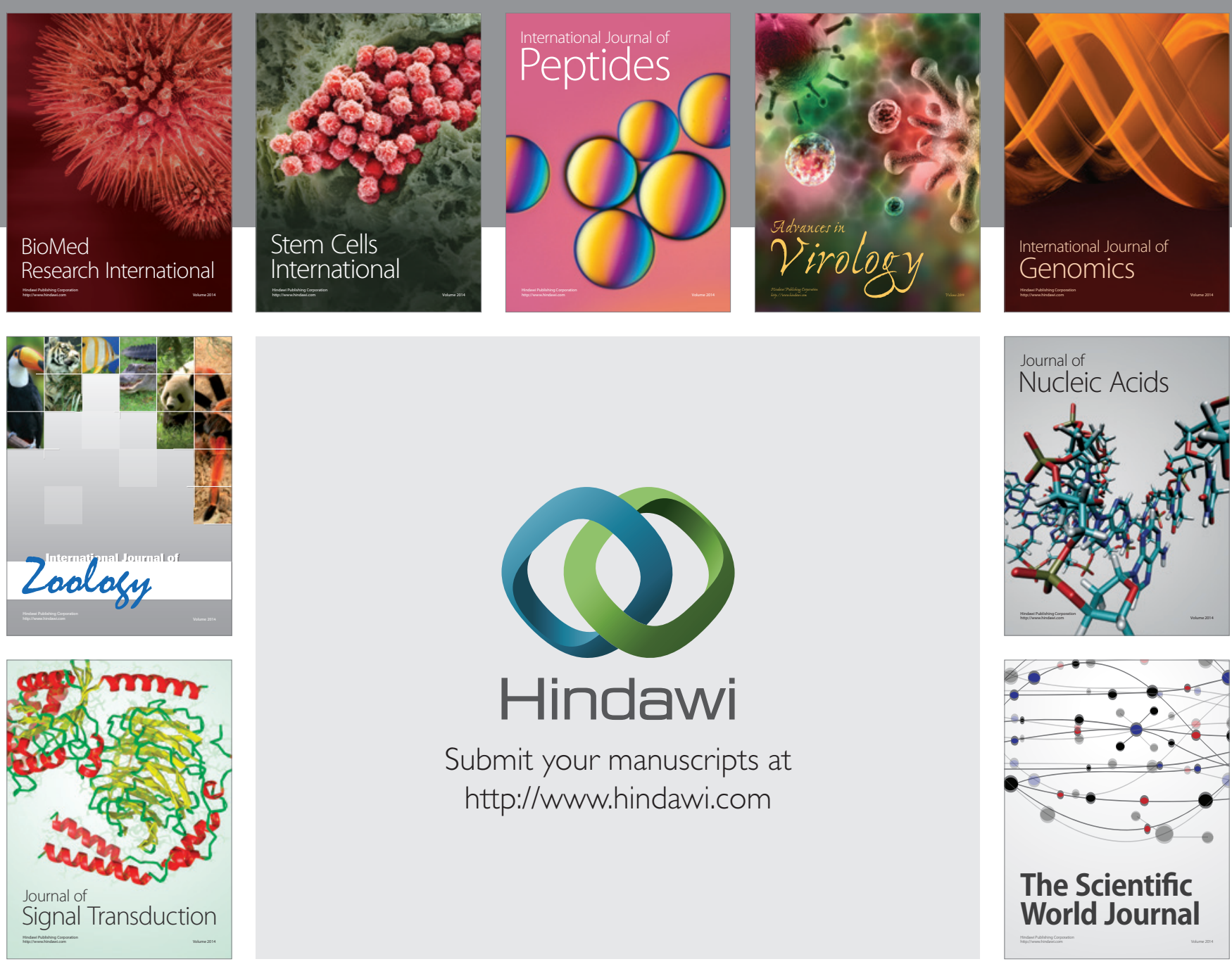

Submit your manuscripts at

http://www.hindawi.com
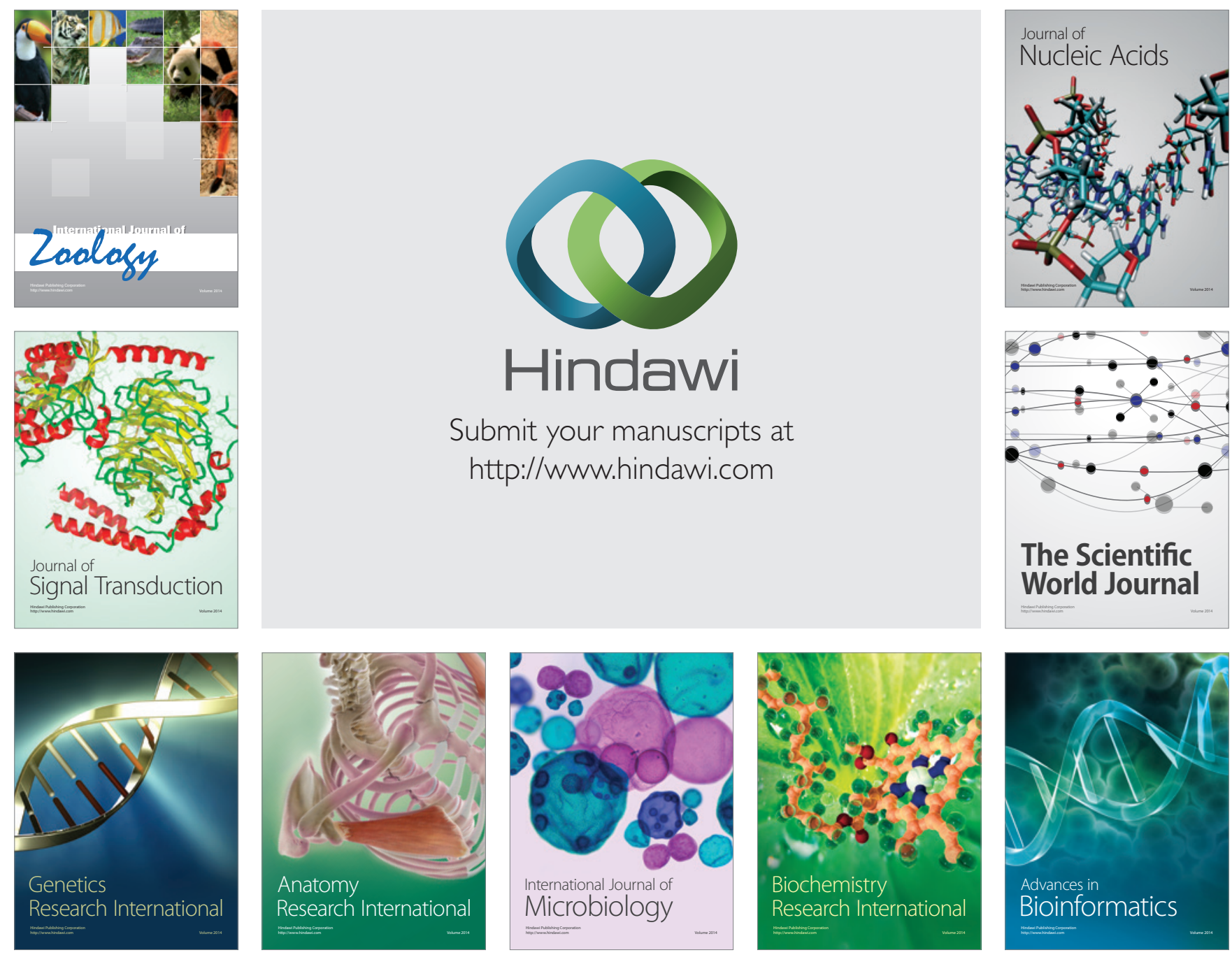

The Scientific World Journal
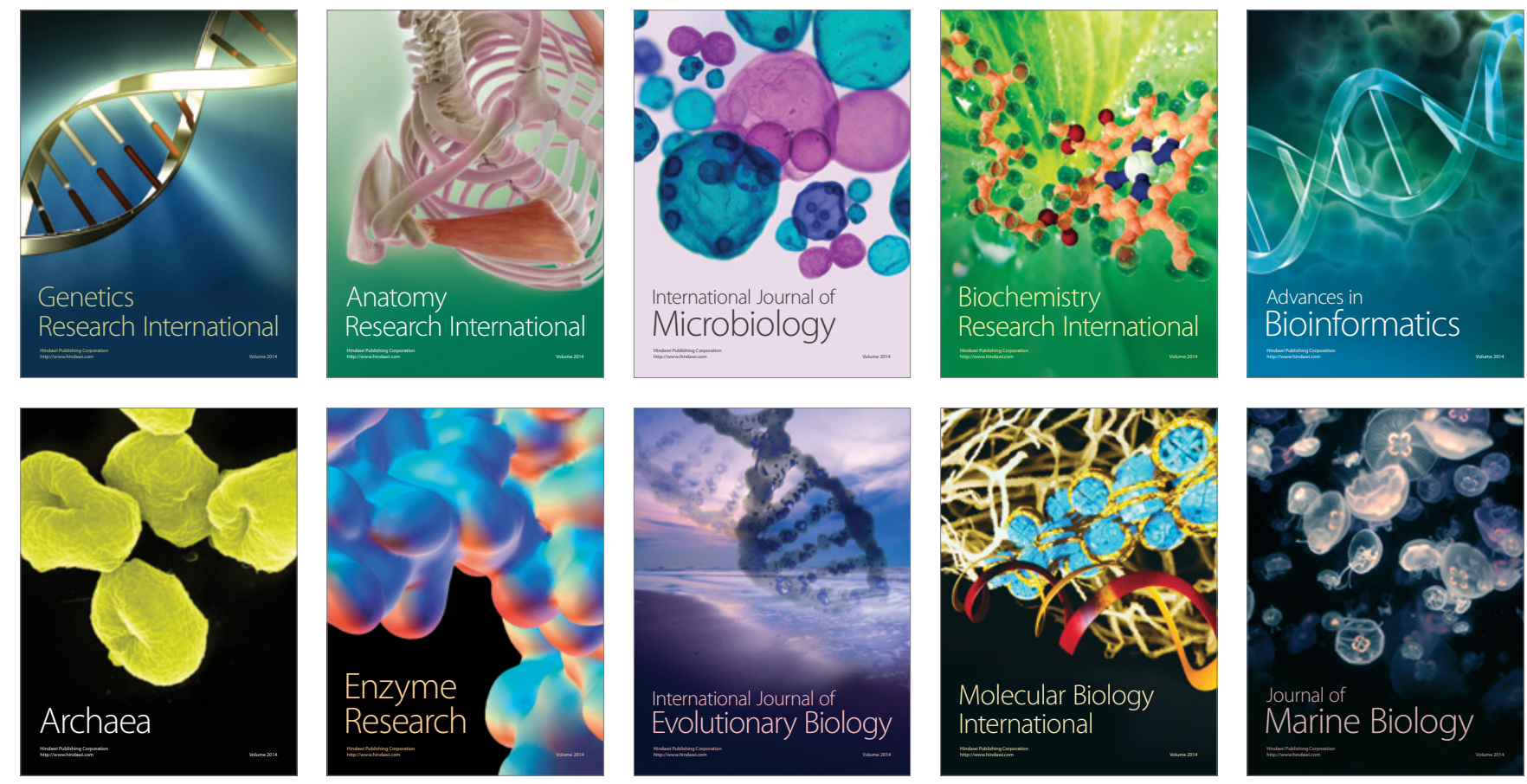